\title{
A General Discerption of Plants' Photosynthetic Pathways iIn Deserts in Saudi Arabia
}

\author{
Ahmed Salem \\ Biology Department, The Northern Borders University, Saudi Arabia \\ Ahmad-ani-27@hotmail.com
}

Abstract: This article aims to know the differences among photosynthesis pathway based on climate conditions in deserts in Saudi Arabia.

\section{INTRODUCTION}

Unlike animals, who are capable of movement, plants rely on photosynthesis to make their own food. Photosynthesis can in general, be defined as the process by which plants (specifically green plants) and some organisms synthesize food from Carbon (IV) oxide and water by utilizing energy from the sun. This simple chemical process, a marvel of nature's engineering, is the basis of life in the entire food chain. (6)

Photosynthesis, like other metabolic processes follows a specific metabolic pathway. Metabolic pathways can be described as the steps that a biochemical reaction follows in the process of converting molecules into readily usable materials. Photosynthetic pathways therefore refer to the steps involved in photosynthesis. For the three types of photosynthesis; C3, C4, and CAM the pathways followed are basically the same. However, the differences among these arise from the way each plant chooses to carry out photosynthesis. The choice of the type of photosynthesis that a plant would carry out is entirely dependent on the environmental conditions that surround a particular plant

Saudi Arabia is predominantly desert with a few areas especially along the coastline and highland regions showing ample signs of flora and fauna. The rest of the region shows scattered populations of herbs, shrubs and trees that are surviving the harsh desert conditions. Saudi Arabia's flora population is mostly concentrated in the mountainous regions which are mostly found in the northwestern. Furthermore, most of these species bear close resemblance to those of East Africa, parts of North Africa and The Mediterranean regions that share similar climatic characteristics to part of Saudi Arabia. (7)

\section{THE PHOTOSYNTHETIC PATHWAY}

As mentioned earlier, a photosynthetic pathway is the steps taken by a plant in the process of converting Carbon (IV) Oxide and water molecules into complex sugars using energy from the sun. The three basic types of photosynthesis are based on two pathways; the light stage reactions and the dark reactions.

During the light reactions, the sun's energy is absorbed into the plant by the chlorophyll. Once this energy has been absorbed, this energy initiates and drives the transfer of electrons and hydrogen ions from the water absorbed by the plant to the NADP+ which stands for nicotine-amide adenine dinucleotide phosphate (4). The $\mathrm{NADP}+$ is an acceptor that stores the energized electrons on a temporary basis. This entire reaction results in the splitting of water molecules and the release of the oxygen atom as a byproduct. Once both the hydrogen nucleus and the electrons reach the NADP+ the convert it to NADPH by adding the two together. The NADPH thus becomes a reducing agent to be used in the dark reactions. The light reactions also result in the formation of ATP (adenosine triphosphate) which stores the chemical energy that will be used in the next stage. 
The dark reactions, also known as the Calvin cycles are the reactions that result in the actual production of the sugars or "food". The process begins by carbon fixation, which is essentially the incorporation or combination of carbon (IV) oxide from the atmosphere into the organic molecules already present in the chloroplasts of the plant cells. The Carbon (IV) oxide is then reduced by the NADPH with the energy in the form of ATP. The final products of this reactions are the sugars and NADP+ and ADP. The sugars are them transported by the phloem vessels to storage while the NADP+ and the ADP are recycled to be used again the light reactions. As had been mentioned earlier the three types will follow these basic pathways however, the difference will come in the details of how the processes are carried out, a difference that can be attributed to the environment of the plant.

\section{C3 photosynthesis}

Also, referred to as normal photosynthesis in some quarters, C3 pathway is by far the most common type of photosynthetic pathway. In extreme cases, C3 plants have been in the coldest arctic habitats and in the warmest deserts. Although in normal condition these types of plants tend to thrive in areas with ample supply of water.

This pathway is called the C3 pathway mainly due to the initial products that are formed at carbon fixation, which are 3-carbon compounds otherwise known as phosphoglyceric acid. During C3 photosynthesis, the carbon (IV) oxide molecules from the carbon fixation are combined with 5-carbon sugars known as ribulose bisphosphate (RuBP) in the presence of the enzyme ribulose bisphosphate carboxylase/oxygenase or in simpler terms rubisco. Rubisco is also responsible for catalyzing the reaction of RuBP with oxygen and leads to photo respiration (3).

Photorespiration occurs when there is too much oxygen present in the plant prompting a reaction with RuBP. Releasing 2-phosphoglycolate which can undergo further metabolism during photorespiration to release Carbon (IV) Oxide in daylight. This process results in net reduction I photosynthesis mostly because of the competition between Carbon (IV) Oxide and oxygen molecules for the active sites of the enzyme rubisco. Once the oxygen molecules populate the active sites of the rubisco, it cannot take up Carbon (IV) Oxide hence hampering the photosynthetic process.(6)

Photorespiration is especially common in regions with higher temperatures or in hot sunny conditions because of the reduced water presence. Under normal circumstances, the oxygen molecules produced during the light reactions exit through the stomata of the leaves. However, with there is little or no water, the plants will tend to open their stomata less frequently to prevent water loss due to photorespiration. The outcome of this is that the oxygen levels may build up within the leaf triggering the competition for the active sites of rubisco therefore leading to photorespiration (3).

\section{C4 Photosynthesis}

The C4 photosynthesis differs greatly from the normal photosynthesis in that C4 takes place in the presence of the enzyme Phosphoenolpyruvate carboxylase also known carboxylase. This enzyme differs from rubisco in that it possesses a greater affinity for Carbon (IV) Oxide than oxygen therefore reducing the possibility of photorespiration.

During C4 photosynthesis, Carbon (IV) Oxide obtained from carbon fixation is reacted with PEP with PEP carboxylase acting as a catalyst. The end result of this process is a 4-carbon acid known as oxaloacetate. The direct consequence of this is that the stomata can be closed for longer periods of time without harming the leaf c4 photosynthesis is therefore the most suited in hot and dry climates (3).

Another distinguishing factor for the $\mathrm{c} 4$ reaction is that the reactions do not take place within the same cells. The primary reactions take place in the outer mesophyll cells that have PEP carboxylase and some chloroplasts. 
Once reactions within this layer is complete, the end products (oxaloacetate) are sent into the bundle sheath cells that surround the xylem and the phloem. The rest of the Calvin cycle take place within these cells.(6)

The c4 photosynthesis however has a few drawbacks. The outer mesophyll layers are continuously pumping carbon into the bundle sheath cells which contain fewer cells in comparison. The result of this is that the ratio of Carbon (IV) Oxide to oxygen remains high within these cells having the positive effect of preventing photorespiration but with the negative effect of low sugar production. Although the C4 respiration is considered inefficient due to continuous carbon pumping, it suffices in low water and/or dry conditions.(6)

\section{THE CAM PHOTOSYNTHESIS}

The Crassulacean Acid Metabolism commonly known as CAM bears a great deal of similarity with the c4 photosynthesis. However, the difference presents itself in the timing of the stomatal opening and closing. In this type of photosynthesis, the stomata remain open at night allowing carbon (IV) Oxide to diffuse into the leaf and react with PEP to form the oxaloacetate acid which is then stored overnight in the central vacuoles. When day breaks, the acid is released and undergoes a process of decarboxylation to release Carbon (IV) Oxide.

The carbon (IV) Oxide released is then combined with RuBP just as it does in c3 photosynthesis. A key characteristics of plants that undergo this type of photosynthesis, is that they usually possess thick and fleshy leaves that are purposed to store water or stems that perform the same function (3). This plants are usually associated with the term succulent.

Another characteristic of these plants is that the stored carbon runs out fast and since photosynthesis only takes place for part of the day, very little sugar is produced. This means that plants that grow under these conditions tend to have very slow growth. The CAM photosynthesis is typical of desert plants, due to their slow growth in this harsh conditions, desert plants have evolved very intricate defense systems to protect against animals that would want to take away such slow gains (2).

\section{Variations in photosynthesis pathways among plants}

The significance of the variations in photosynthesis pathways is that it enables plants to better adapt and perform in their different environments. Among the three pathways, C3 is the most common and widespread found in a variety of environments ranging from warm deserts to cold arctic habitats. C4 pathway is the second most common pathway found mostly in grass species. It is also found in vascular plant species. These plants tend to thrive in sunny and warm climates with relatively low water abundance. CAM photosynthesis is typical of plants in desert conditions and are characterized by plants with larger vacuoles (3). They key disadvantage of this pathway is that the plants involved tend to have low sugar producing capabilities, very slow growth rates and are poor competitors (although they have little to no competition).

\section{Factors that affect photosynthetic pathways}

The photosynthesis pathways described above have one thing in common. They are all enzyme dependent. That is to say, for photosynthesis to successful, the enzymes must be working in the right conditions and rate with near $100 \%$ efficiency. However, this ideal situation is impossible to achieve since environment varies from place to place and is ever evolving. The main environmental conditions that affect photosynthesis are; temperature and soil ph.

\section{TEMPERATURE}

Enzymes are primarily made up of proteins and as such are highly sensitive to changes in temperature. The operating temperature of most enzymes is maximum at 40 degrees Celsius and minimum just above freezing. At higher temperatures, the structure of the enzyme is destroyed hence affecting the rate by which these enzymes function. 
However, an increase in temperature would result in a corresponding increase in rate of photosynthesis. This is so because the higher temperatures provide the enzymes with enough energy hence allowing them to collide more with their substrates. The reverse is also true in this particular case; the lower the temperature the slower the rate of photosynthesis, this is so because the enzymes do not possess enough energy to allow them continuously with the substrate. At temperatures nearing freezing, the enzymes of some plants completely reduce or stop altogether(4)

\section{Soil ph}

In simple terms, ph. Can be defined as the acidity or alkalinity of a substance. The ph. of a soil therefore refers to the acidity or alkalinity of soil in a particular area. It all still trickles down to structure. Different enzymes have different levels of tolerance to both high and low ph. however, each plant species have their own level of tolerance to acidity or alkalinity which determine its optimum operation. If this optimum level is exceeded, then photosynthesis rate will most likely reduce.(8)

\section{Topography and climate}

Saudi Arabia, which is one of the largest countries in the world, is home to the world's largest continuous sand desert the al-Rub al-Khali or the Empty Quarter as it is otherwise known. The other major regions of Saudi Arabia are; The Najd, the Hijaz and the eastern province. The Najd, which is by far the largest, is a central plateau rising to about 600 meters above sea level on its eastern side and about 1,500 meters above sea level on its western side. This region has political significance as it is where the capital, Riyadh, is located. The other region, the Hijaz stretches from the Gulf of Aqaba south to the regions of Asir, this area has religious significance as it is where the holy cities of Mecca and Medina are located. The areas around Asir also contain a fertile coastal plain (2). The Asir region also contain inland mountains that have been estimated to rise to about 2,700 meters above sea level. The other major region, the Eastern Province, is located along the Arabian Gulf and contains most of the country's oil reserves. Apart from the oil reserves, the eastern province also comprises of oases occupying most of the al-Ahsan region. (7)

The climate of Saudi Arabia can generally be described as hot and dry, with very high daytime temperatures reaching up to 45 degrees Celsius and cooler night time temperatures. The daytime temperatures are relatively lower in spring and autumn reaching up to 29 degrees Celsius. Night time temperatures have been recorded below 0 degrees in winter. This climate has been mostly observed in the central plateaus of the Najd, the al-Qassim province and around the surrounding deserts. The maximum daily temperatures along the Saudi coastlines average a constant of about 38 degrees. These areas run along the Red Sea and the Arabian Gulf (1). The regions that are elevated, such as Asir and lower Hijaz which rise to about 3,000 meters above sea level tend to be cooler, with maximum temperatures below 35 degrees. These regions experience the most rainfall in Saudi Arabia, most of which fall during the monsoon seasons, autumn and spring. The average rainfall recorded in this region range from about 250 to 300 millimeters. Making this area the most suitable for vegetation to. The other Saudi Arabian regions receive very little rainfall throughout the year limiting the vegetation to only a few shrubs and baobabs scattered along the deserts. (7)

\section{Plant distribution in Saudi Arabia}

As has been described above, most of the Saudi environment is made up of harsh desert with little to none rainfall throughout the year. The areas mostly comprise of the northern, eastern and the central areas. These regions are populated, although sparsely, with trees like the acacias and a variety of wild shrubs and herbs. Succulent plants such as the cactus are also reported in some regions of Saudi Arabia (4).

However, most vegetation is found along the northwestern and southwestern regions of Saudi Arabia especially around the mountainous regions of Asir and Hijaz and along the fertile coastal plains of Asir along the Arabian Gulf (4). These areas receive the highest amount of rainfall due to the high elevations and the monsoon winds from the Indian Ocean.

Volume 3

Page 4 
The predominantly hot and dry climates of Saudi Arabia especially in the northern and central regions, favor plants that undergo the CAM photosynthesis. This plants open their stomata at night to let in carbon (IV) Oxide that the fuse with PEP to form a malate. This then undergoes decarboxylation to release Carbon(IV)oxide that combine with RuBP in the normal c3 pathway hence the plant is able to manufacture its food in the normal way when day breaks. This adaptation allows these desert plants to survive the harsh desert conditions (2). Furthermore, these extreme plants also known as xerophytes, also possess succulent stems and leaves, these are vital for storage of food and water. (8)

C4 photosynthesis is also common in plants in these areas. Although these plants tend to favor the semi-arid areas around the northwest and southwest which receive considerable amounts of rainfall. It should be noted however that although these regions receive more rainfall than the rest of the Arabian Peninsula, these rainfall is much lower than those found in the tropical regions. Therefore, most vegetation has adapted to this photosynthesis pathway, however inefficient, to enable them survive the short water supply and the prolonged stomatal closures that help prevent evapotranspiration. (9) (10)

Therefore, when deciding on the areas where desert plants can be planted and easily monitored, the most recommended sites would be the southern coastal plains due to their fertility and consistent rainfall. The elevated areas of Asir and Hijaz are also likely candidates mostly due to their lower temperatures and rainfall presence. The mountainous regions of Asir are also a site to be considered although access may prove to be difficult.

\section{REFRENCES}

Almazroui, M., Nazrul Islam, M., Athar, H., Jones, P.D., \& Rahmab, M. A. (2012). Recent climate change in the Arabian Peninsula: annual rainfall and temperature analysis of Saudi Arabia for 1978-2009. International journal of climatology, 32(6), 953-966

El-Demerdash, M. A., Hegazy, A. K., \& Zilay, A. M. (1994). Distribution of the plant communities in the Tihamah coastal plains of Jazan region, Saudi Arabia. Journal of Vegetation and Distribution, 112(2), 141-151

Ellis, R., Vogel, J. C., \& Fuls, A. (1980). Photosynthetic pathways and the geographical distribution of grasses in South West Africa/Namibia. South African Journal of Science, 76(7), 307-314.

Ziegler, H., Batanouny, K. H., Sankhla, N., Vyas, O. P., \&Stichler, W. (1981). The photosynthetic pathway types of some desert plants from India, Saudi Arabia, Egypt and Iraq. Oecologia, 48(1), 93-99

"Photosynthesis: An Overview." Photosynthesis Advances in Photosynthesis and Respiration(n.d.): 1-46. Web.

Gowik, U., and P. Westhoff. "The Path from C3 to C4 Photosynthesis." Plant Physiology 155.1 (2010): 56-63 Web.

Mcclure, H. A. "Ar Rubâ Al Khali." Quaternary Period in Saudi Arabia (1978): 252-63. Web.

Koyro, Hans -Werner. "Special Issue on Learning from Extremophytes: Xerophytes and Halophytes." Emirates Journal of Food and Agriculture 26.12 (2014): n. pag. Web.

Edwards, Gerald E., and Elena V. Voznesenskaya. "Chapter 4 C4 Photosynthesis: Kranz Forms and Single-Cell C4 in Terrestrial Plants." C4 Photosynthesis and Related CO2 Concentrating Mechanisms Advances in Photosynthesis and Respiration (2010): 29-61. Web.

Arabian Peninsula (Saudi Arabia, Kuwait, Bahrain, Qatar, United Arab Emirates, Oman, North \& South Yemen). Washington, D.C.: Naval Personnel Command, 1981. Print.

Citation: Ahmed Salem, "A General Discerption of Plants' Photosynthetic Pathways iln Deserts in Saudi Arabia". American Research Journal of Biosciences; Volume 3, 2017; pp:1-5

Copyright (c) 2017 Ahmed Salem, This is an open access article distributed under the Creative Commons Attribution License, which permits unrestricted use, distribution, and reproduction in any medium, provided the original work is properly cited.

Volume 3

Page 5 